\title{
Development of a New Index Based on Preoperative Serum Lipocalin 2 to Predict Post-LSG Weight Reduction
}

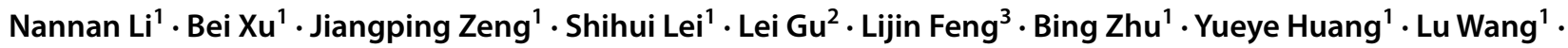 \\ Lili $\mathrm{Su}^{1} \cdot$ Shen $\mathrm{Qu}^{1} \cdot$ Xiaoyun Cheng ${ }^{1} \cdot$ Le Bu$^{1} \mathbb{D}$
}

Received: 16 November 2021 / Revised: 3 January 2022 / Accepted: 14 January 2022 / Published online: 9 February 2022

(c) The Author(s) 2022

\begin{abstract}
Background Bariatric surgery is the most effective therapy for obesity, but targeted weight reduction is not always achieved. Serum lipocalin-2 (LCN2) is closely associated with obesity, but its impact on weight loss after surgery is unknown. We aimed to access the reliability of LCN2 levels and other parameters as effective predictors of excellent weight loss ( $\geq 75 \%$ excess weight loss (EWL)) 1 year after bariatric surgery.

Methods This retrospective study evaluated 450 patients (aged 18-65 years) with obesity at 3 months and 1 year after laparoscopic sleeve gastrectomy (LSG) surgery. Seventy-four patients who underwent LSG surgery and met the inclusion and exclusion criteria were included in this study. Serum LCN2, thyroid function, and metabolic and anthropometric parameters were assessed. Weight reduction was expressed as \%EWL and percent total weight loss (\%TWL) at 3 months and 1 year post surgery. Multivariable logistic regression analysis and receiver operating characteristic (ROC) curve analysis were used to evaluate predictors of $\geq 75 \% \mathrm{EWL}$.

Results In our cohort, \%EWL and \% TWL were both strongly associated with preoperative serum LCN2 levels. The binary logistic regression analysis showed that preoperative LCN2, waist circumference, and glycated hemoglobin were independent predictors of excellent weight loss.

Conclusions Based on these results, we determined a new $\mathrm{P}$ index with better predictive value for excellent weight reduction $(\geq 75 \%$ EWL) 1 year after LSG surgery.
\end{abstract}

Keywords Lipocalin-2 $\cdot$ Bariatric surgery $\cdot$ Obesity $\cdot$ Weight loss

\section{Key points}

- Excess- and total-weight loss were associated with pre-op serum lipocalin-2 levels

- Pre-op lipocalin-2, waist circumference, and glycated hemoglobin were correlated with excess weight loss

- The newly developed index, $P=(-0.017 \times$ age

(years) $-2.134 \times$ sex $-0.014 \times$ BMI $\left(\mathrm{kg} / \mathrm{m}^{2}\right)-0.266 \times$ waist circumference $(\mathrm{cm})+0.073 \times \mathrm{LCN} 2(\mathrm{ng} /$

$\mathrm{mL})+0.566 \times$ triglycerides $(\mathrm{mmol} / \mathrm{L})-0.741 \times \mathrm{HbAc} 1$

$(\%)+33.018)$, with sex referred to as 1 in men and 2 in women.

- A cut-off point of 0.649 predicted excellent weight loss

12 -month post-op with a sensitivity and specificity above $80 \%$

Nannan Li and Bei Xu have contributed equally to thiswork.

Xiaoyun Cheng

chengxiaoyun0210@163.com

$\triangle$ Le Bu

geyingjun@hotmail.com

\section{Introduction}

Obesity is associated with many severe comorbidities and has developed into an ongoing pandemic [1]. Bariatric surgery is an effective therapy for rapid, substantial, and durable weight loss, while improving metabolism and reducing mortality and morbidity [2-6]. However, not all bariatric surgery patient reach the expected effective weight loss due to multiple factors [7], such as socioeconomic status,

2 Department of Gastrointestinal Surgery, Shanghai Tenth People's Hospital, Tongji University School of Medicine, No. 301 Middle Yanchang Road, Shanghai 200072, China

3 Department of Pathology, Shanghai Tenth People's Hospital, Tongji University School of Medicine, No. 301

Middle Yanchang Road, Shanghai 200072, China

1 Department of Endocrinology and Metabolism, Shanghai Tenth People's Hospital, Tongji University School of Medicine, No. 301 Middle Yanchang Road, Shanghai 200072, China 
operation style, sex, and preoperative characteristics, which have been studied previously.

Recently, myriad of studies have devoted considerable attention to lipocalin-2 (LCN2) for its potential as a novel therapeutic target for obesity. LCN2 is a new adipokine primarily secreted by osteoblasts and adipocytes. Elevated serum LCN2 levels have been found in obesity models in mice and humans [8, 9]. Furthermore, the involvement of LCN2 might result in the deceleration of spontaneous agerelated adiposity by the browning of white adipose tissue and promotion of thermogenic and mitochondrial activity in mice [10]. As reported by Petropoulou et al., LCN2 is a centrally acting anorexigenic hormone that is conserved in humans and non-human primates [11].

These observations revealed that LCN2 plays an indispensable role in obesity. However, the relationship between LCN2 and the remission of obesity after bariatric surgery remain unclear. Our study explored the association of weight reduction after laparoscopic sleeve gastrectomy (LSG) with serum LCN2 levels or other preoperative factors. We propose a new $\mathrm{P}$ index to be used as an easy "rule of thumb" for predicting excellent weight reduction after bariatric surgery.

\section{Materials and Methods}

\section{Subjects}

This retrospective study evaluated 450 patients (aged 18-65 years) with obesity who underwent LSG at Shanghai Tenth People's Hospital between July 2018 and July 2021. The study recruitment flow chart is shown in Fig. 1. Key inclusion criteria included (1) body mass index (BMI) of $32.5 \mathrm{~kg} / \mathrm{m}^{2}$ or higher or (2) $27.5 \mathrm{~kg} / \mathrm{m}^{2}$ or higher BMI with no less than two obesity-related comorbid conditions (e.g., type 2 diabetes, hypertension, dyslipidemia, and obstructive sleep apnea) [9, 12, 13]. Key exclusion criteria included missing data on the required parameters (anthropometric or metabolic data), previous bariatric procedures, use of psychiatric medications or steroids known to affect body weight, severe diseases (malignancy, connective tissue diseases, endocrine diseases, end-stage cardiac, hepatic, or renal failure), and uncontrolled psychiatric diseases $[14,15]$. Seventy-four patients who underwent LSG and met the inclusion and exclusion criteria were

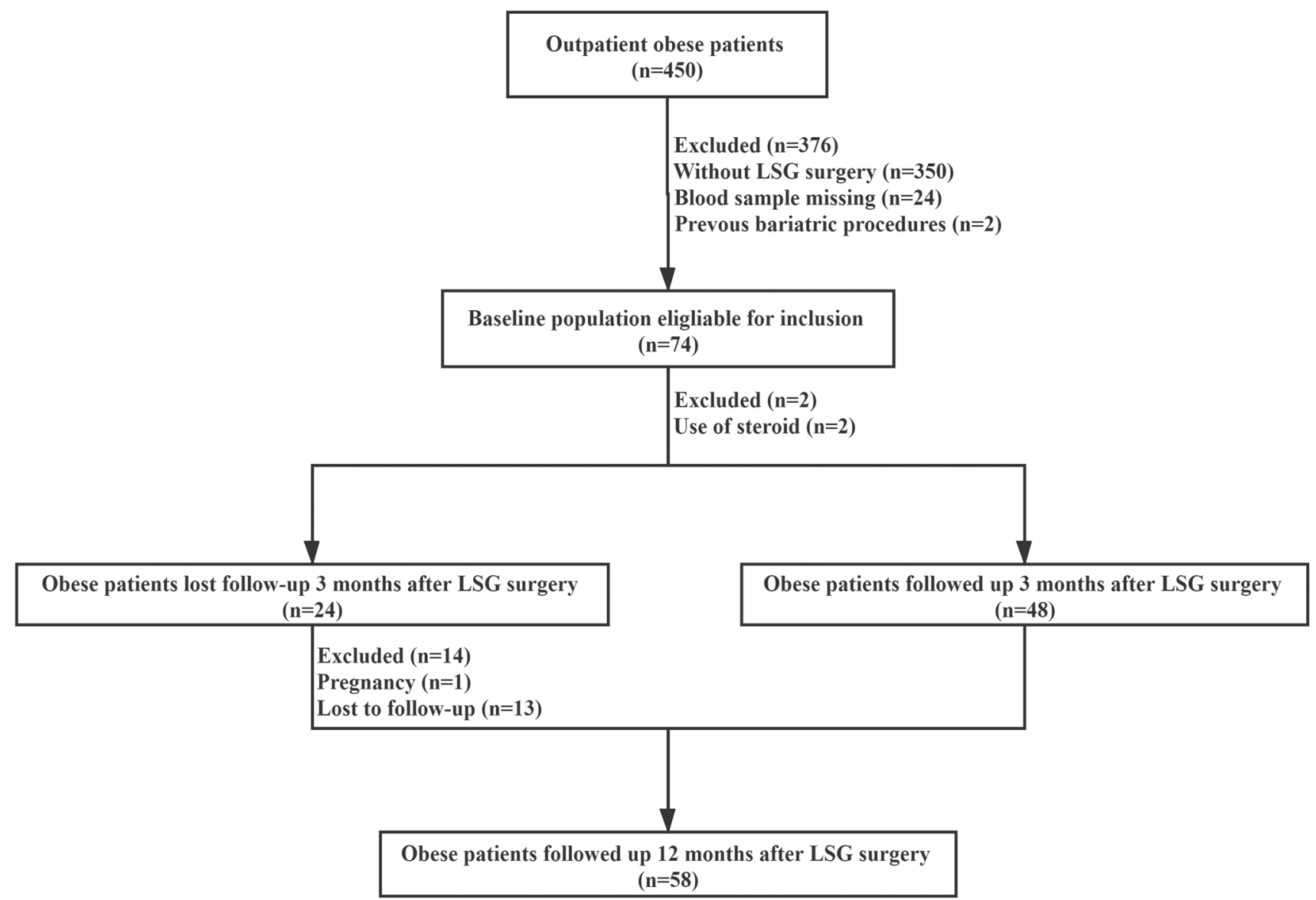

Fig. 1 Follow-up study flow chart on patients with obesity subjected to laparoscopic sleeve gastrectomy (LSG) surgery at 3 and 12 months 
recruited for this study (Fig. 1). Of the 74 patients, 48 (64.9\%) completed follow-up at 3 months and 58 (78.4\%) completed follow-up at 12 months after LSG.

The study was approved by the ethics committee of Shanghai Tenth People's Hospital. All patients provided written informed consent.

\section{Evaluation of Anthropometric Parameters}

Sex, age, body weight, BMI, neck circumference, waist circumference (WC), hip circumference, and blood pressure were evaluated as preoperative parameters on standard medical scales. Weight loss was evaluated by percent excess weight loss (\%EWL) and percent total weight loss (\% TWL), with the primary outcome of the study being \%EWL 1 year after LSG. \%EWL after 3 months and 1 year follow-up was calculated by the formula: ((preoperative weight - weight at third / 12th month) / (preoperative weight - ideal weight to produce BMI $\left.24 \mathrm{~kg} / \mathrm{m}^{2}\right) \times 100$ ) [16]; \%TWL after 3 months and 1 year follow-up was defined by the formula: ((preoperative weight - weight at third / 12th month) / (preoperative weight $\times 100$ ). According to Reinhold's classification [17, 18], insufficient weight loss after LSG surgery was regarded as $<50 \%$ EWL, while excellent weight loss was defined when the $\%$ EWL was $\geq 75 \%$. Hypertension was defined as blood pressure $\geq 140 / 90 \mathrm{mmHg}$, or previously physiciandiagnosed and treated [7].

\section{Measurement of Metabolic Parameters}

The serum levels of triglycerides (TG), total cholesterol (TC), free fatty acid (FFA), high-density lipoprotein (HDL) cholesterol, low-density lipoprotein (LDL) cholesterol, glycated hemoglobin $(\mathrm{HbAlc})$, fasting plasma glucose (FPG), and 2-h postprandial plasma glucose (2hPG) were measured and recorded at baseline. Diabetes was defined by $\mathrm{FPG} \geq 7.0 \mathrm{mmol} / \mathrm{L}, \mathrm{HbA} 1 \mathrm{c} \geq 6.5 \%, 2 \mathrm{hPG} \geq 11.1 \mathrm{mmol} / \mathrm{L}$, or previously diagnosed as diabetic and treated; Dyslipidemia was defined by $\mathrm{LDL} \geq 4.14 \mathrm{mmol} / \mathrm{L}, \mathrm{HDL}<1.04 \mathrm{mmol} / \mathrm{L}$, $\mathrm{TG} \geq 2.26 \mathrm{mmol} / \mathrm{L}$, or previously diagnosed and treated [7]. Thyroid-stimulating hormone (TSH), free triiodothyronine (FT3), free thyroxine (FT4), total triiodothyronine (TT3), and total thyroxine (TT4) levels were evaluated to determine preoperative thyroid function in patients with obesity.

\section{ELISA for Serum LCN2 Measurements}

Serum LCN2 levels were measured preoperatively using Human Lipocalin-2/NGAL Quantikine ELISA Kit (R\&D Systems, Catalog \#DLCN20; RRID: AB_2894833). The within-assay (percent coefficient of variation: $3.1-4.4 \%$ ) and between-assay (5.6-7.9\%) variability showed a high level of assay precision.

\section{Statistics Analysis}

Data are presented as mean \pm standard deviation (SD) for continuous variables and as percentages for categorical variables. The correlations between serum LCN2 levels and other variables at baseline were assessed using a partial correlation analysis. We conducted Pearson's or Spearman's correlation analysis between preoperative variables and \%EWL/\% TWL to confirm the eligibility for inclusion in the multivariable linear regression analysis, by which the independent factors were determined. We then performed binary logistic regression in Models 1 and 2 to identify independent predictors related to excellent weight loss after LSG surgery, which was generally defined as $\geq 75 \%$ EWL 1 year after LSG surgery. Model 1 incorporated all independent factors and adjusted for potential confounding factors, including sex, age, and preoperative BMI; Model 2 included all the covariates in Model 1 and preoperative HbAc1 and TG levels. Ultimately, the receiver operating characteristic (ROC) curve was performed to test the prediction of preoperative LCN2 or waist circumference alone, and Model 2 for the binary outcome, determining " $\geq 75 \%$ EWL" or " $<75 \%$ EWL" 1 year after LSG surgery, and further to identify the optimum cutoff according to the largest Youden index. Statistical significance was set at $P<0.05$. All statistical analyses were performed using the SPSS Statistics software version 20.0 .

\section{Results}

\section{Baseline Characteristics and Correlation of Preoperative LCN2 Levels with Baseline Variables}

Of the 74 subjects (50 female and 24 male) who were included in this study, the average age, baseline BMI, and sex were 32.05 years and $38.69 \mathrm{~kg} / \mathrm{m}^{2} ; 67.60 \%$ female, respectively (Table 1 ). The baseline population consisted of subjects who were diagnosed with dyslipidemia (66.20\%), hypertension (37.80\%), and diabetes (54.10\%). All subjects exhibited normal thyroid function and the mean serum LCN2 level was $107.50 \mathrm{ng} / \mathrm{mL}$.

After adjustment for sex and age, we observed that preoperative LCN2 levels were positively associated with BMI, weight, waist circumference, and hip circumference (Table 1). A negative correlation was observed between serum LCN2 levels and total cholesterol at baseline. Notably, even after adjusting for BMI, LCN2 remained 
Table 1 Clinical characteristic of 74 subjects and correlation between serum lipocalin-2 levels and other parameters

\begin{tabular}{llllrl}
\hline Characteristics & value & $r 1$ & $P 1$ & $r 2$ & $P 2$ \\
\hline Lipocalin-2, ng/mL & $107.50 \pm 41.49$ & - & - & - & - \\
Age, years & $32.05 \pm 9.90$ & - & - & - & - \\
Gender female $(n, \%)$ & $50.00,67.60$ & - & - & - & - \\
Body mass index, kg/m ${ }^{2}$ & $38.69 \pm 5.96$ & 0.275 & $0.019^{*}$ & - & - \\
Weight, kg & $110.84 \pm 25.44$ & 0.329 & $0.005^{* *}$ & 0.197 & 0.100 \\
Neck circumference, cm & $41.92 \pm 4.37$ & 0.038 & 0.752 & -0.162 & 0.176 \\
Waist circumference, cm & $118.22 \pm 14.71$ & 0.285 & $0.015^{*}$ & 0.108 & 0.370 \\
Hip circumference, cm & $121.46 \pm 12.65$ & 0.256 & $0.030^{*}$ & 0.036 & 0.767 \\
Hypertension $(n, \%)$ & $28.00,37.80$ & - & - & - & - \\
Dyslipidemia $(n, \%)$ & $49.00,66.20$ & - & - & - & - \\
Triglycerides, mmol/L & $2.21 \pm 1.38$ & -0.078 & 0.515 & -0.028 & 0.815 \\
Total cholesterol, mmol/L & $4.80 \pm 1.01$ & -0.254 & $0.032^{*}$ & -0.251 & $0.034^{*}$ \\
Free fat acid, mmol/L & $0.92 \pm 2.18$ & -0.124 & 0.300 & -0.234 & $0.049^{*}$ \\
HDL cholesterol, mmol/L & $1.02 \pm 0.24$ & 0.063 & 0.597 & 0.101 & 0.404 \\
LDL cholesterol, mmol/L & $2.76 \pm 0.82$ & -0.177 & 0.138 & -0.164 & 0.171 \\
Diabetes $(n, \%)$ & $40.00,54.10$ & - & - & - & - \\
HbA1c, \% & $7.04 \pm 2.12$ & 0.175 & 0.142 & 0.167 & 0.165 \\
Fasting plasma glucose, mmol/L & $6.98 \pm 2.82$ & 0.120 & 0.315 & 0.089 & 0.463 \\
2-h plasma glucose, mmol/L & $11.13 \pm 5.25$ & 0.068 & 0.571 & 0.068 & 0.575 \\
Free T3, pmol/L & $5.04 \pm 0.62$ & 0.028 & 0.818 & 0.005 & 0.964 \\
Free T4, pmol/L & $16.60 \pm 2.29$ & 0.125 & 0.294 & 0.119 & 0.323 \\
Total T3, nmol/L & $1.79 \pm 0.37$ & 0.116 & 0.332 & 0.019 & 0.876 \\
Total T4, nmol/L & $105.86 \pm 21.10$ & 0.123 & 0.305 & 0.077 & 0.525 \\
TSH, mIU/L & $2.84 \pm 3.19$ & 0.005 & 0.967 & 0.007 & 0.951 \\
\hline & & & & & - \\
\hline
\end{tabular}

1 adjusted for sex and age. 2 adjusted for sex, age, and BMI. $* P<0.05$; ** $P<0.01$; *** $P<0.001$ significantly negatively correlated with total cholesterol and free fatty acids.

\section{Association of Weight Reduction with Preoperative LCN2 and Other Parameters}

Forty-eight patients completed a 3-month follow-up and retained a mean loss of $54.76 \%$ of their excess weight and $19.09 \%$ of their total weight (Fig. 2). One year after surgery, the patients' weight loss reached a peak of $79.86 \%$ EWL and $28.68 \%$ TWL. As shown in Table 2, all five baseline anthropometric parameters (weight, BMI, neck, waist, and hip circumference) were negatively correlated with \%EWL at the 3-and 12-month follow-up visits. Free fatty acid and total T3 levels were also negatively correlated with \%EWL.

Preoperative LCN2 levels were positively associated with both \%EWL and \%TWL 3-month and 1-year after surgery (Table 2). As summarized in Table 3, multiple linear regression analysis showed that baseline BMI and serum LCN2 levels were independently associated with \%EWL at 3-month post-surgery. Furthermore, waist circumference and serum LCN2 levels were independently correlated with $\% \mathrm{EWL}$ at the first-year follow-up visit.

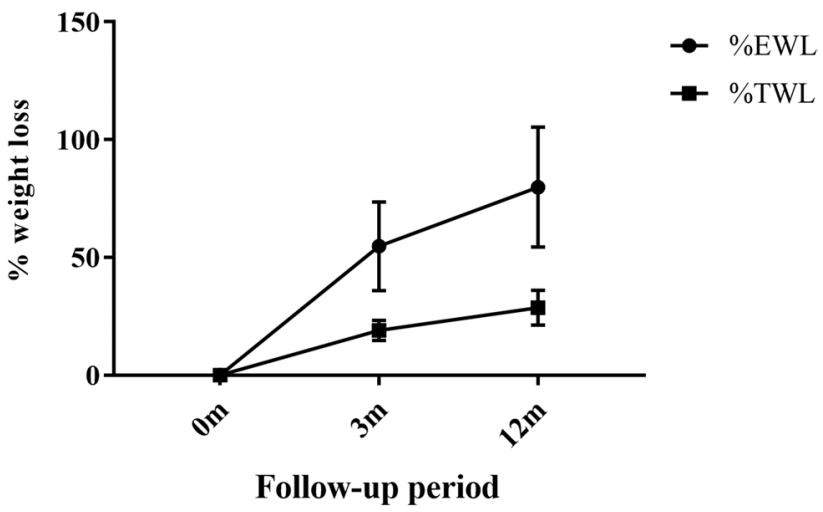

Fig. 2 Weight loss plot over time. Values are shown as the mean values of \%EWL by the circle dots and \%TWL by the square dots, and standard deviation of both by vertical lines. Percent extra weight loss (\%EWL) was calculated by the formula: ((preoperative weight - current weight) / (preoperative weight-ideal weight to produce BMI $\left.24 \mathrm{~kg} / \mathrm{m}^{2}\right) \times 100$ ); percent total weight loss (\%TWL) was defined by the formula: ((preoperative weight-current weight) / (preoperative weight $) \times 100$ ). $0 \mathrm{~m}$ baseline, $3 \mathrm{~m} \mathrm{3}$-month follow-up after laparoscopic sleeve gastrectomy (LSG) surgery, $12 \mathrm{~m} \mathrm{12-month} \mathrm{follow-up}$ after LSG surgery 
Table 2 Associations of weight reduction parameters during follow-up with pre-operative anthropometric and metabolic parameters

\begin{tabular}{|c|c|c|c|c|c|c|c|c|}
\hline \multirow[t]{3}{*}{ Characteristics } & \multicolumn{4}{|l|}{3 months } & \multicolumn{4}{|c|}{12 months } \\
\hline & \multicolumn{2}{|c|}{$\%$ EWL, \% } & \multicolumn{2}{|c|}{$\% \mathrm{TWL}, \%$} & \multicolumn{2}{|c|}{$\%$ EWL, \% } & \multicolumn{2}{|c|}{$\%$ TWL, \% } \\
\hline & - & $P$ & - & $P$ & - & $P$ & - & $P$ \\
\hline Weight, kg & -0.625 & $<0.001^{* * * *}$ & -0.139 & 0.347 & -0.508 & $<0.001^{* * *}$ & 0.029 & 0.831 \\
\hline Body mass index, $\mathrm{kg} / \mathrm{m}^{2}$ & -0.806 & $<0.001^{* * *}$ & -0.187 & 0.203 & -0.649 & $<0.001^{* * *}$ & 0.031 & 0.816 \\
\hline Neck circumference, $\mathrm{cm}$ & -0.431 & $0.002^{* *}$ & -0.208 & 0.155 & -0.400 & $0.002^{* *}$ & -0.061 & 0.650 \\
\hline Waist circumference, $\mathrm{cm}$ & -0.647 & $<0.001^{* * *}$ & -0.175 & 0.235 & -0.631 & $<0.001^{* * *}$ & -0.101 & 0.451 \\
\hline Hip circumference, $\mathrm{cm}$ & -0.698 & $<0.001^{* * *}$ & -0.244 & 0.094 & -0.592 & $<0.001^{* * *}$ & -0.053 & 0.693 \\
\hline Triglycerides, mmol/L & 0.106 & 0.472 & 0.008 & 0.956 & -0.002 & 0.986 & -0.054 & 0.689 \\
\hline Total cholesterol, mmol/L & -0.004 & 0.977 & 0.019 & 0.896 & 0.033 & 0.803 & 0.101 & 0.453 \\
\hline Free fat acid, mmol/L & -0.461 & $<0.001^{* * *}$ & -0.176 & 0.230 & -0.330 & $0.011^{*}$ & 0.059 & 0.661 \\
\hline HDL cholesterol, $\mathrm{mmol} / \mathrm{L}$ & 0.155 & 0.294 & 0.071 & 0.634 & 0.162 & 0.226 & 0.098 & 0.464 \\
\hline LDL cholesterol, $\mathrm{mmol} / \mathrm{L}$ & -0.087 & 0.557 & -0.074 & 0.615 & 0.052 & 0.700 & 0.153 & 0.250 \\
\hline HbA1c, $\%$ & -0.039 & 0.794 & -0.009 & 0.951 & -0.086 & 0.520 & -0.039 & 0.769 \\
\hline Fasting plasma glucose, $\mathrm{mmol} / \mathrm{L}$ & -0.010 & 0.949 & -0.004 & 0.980 & -0.070 & 0.604 & -0.026 & 0.849 \\
\hline 2-h plasma glucose, $\mathrm{mmol} / \mathrm{L}$ & 0.091 & 0.536 & 0.027 & 0.858 & -0.031 & 0.820 & -0.066 & 0.625 \\
\hline Free T3, pmol/L & -0.062 & 0.675 & 0.044 & 0.766 & -0.034 & 0.800 & 0.074 & 0.580 \\
\hline Free T4, pmol/L & 0.049 & 0.742 & -0.075 & 0.611 & -0.067 & 0.617 & -0.121 & 0.365 \\
\hline Total T3, nmol/L & -0.371 & $0.009^{* *}$ & -0.204 & 0.164 & -0.268 & $0.042^{*}$ & -0.045 & 0.739 \\
\hline Total T4, nmol/L & -0.019 & 0.899 & 0.031 & 0.834 & -0.034 & 0.801 & 0.062 & 0.642 \\
\hline TSH, mIU/L & -0.053 & 0.722 & 0.003 & 0.986 & 0.032 & 0.811 & -0.064 & 0.631 \\
\hline Lipocalin-2, ng/mL & 0.356 & $0.013^{*}$ & 0.546 & $<0.001^{* * * *}$ & 0.395 & $0.002^{* *}$ & 0.493 & $<0.001^{* * * *}$ \\
\hline
\end{tabular}

$\% E W L$ percent extra weight loss, $\% T W L$ percent total weight loss. $* P<0.05 ; * * P<0.01 ; * * * P<0.00$

Table 3 Multiple linear regression of percent excess weight loss vs. demographic and clinical variables

\begin{tabular}{llcccc}
\hline \%EWL (\%) & \multicolumn{2}{c}{3 months $\left(R^{2}=0.710\right)$} & \multicolumn{2}{l}{$\begin{array}{l}12 \text { months } \\
\left(R^{2}=0.614\right)\end{array}$} \\
\cline { 2 - 3 } \cline { 5 - 6 } & \multicolumn{2}{c}{$P$ value } & & \multicolumn{2}{c}{$P$ value } \\
\hline Age & -0.095 & 0.320 & & 0.011 & 0.907 \\
Sex (female vs. male) & -0.043 & 0.686 & -0.055 & 0.616 \\
Body mass index & -0.624 & $<0.001^{* * *}$ & -0.302 & 0.072 \\
Waist circumference & -0.176 & 0.308 & & -0.449 & $0.015^{*}$ \\
Free fat acid & 0.146 & 0.142 & & 0.059 & 0.556 \\
Total T3 & -0.097 & 0.343 & 0.036 & 0.729 \\
Lipocalin-2 & 0.385 & $<0.001^{\text {*** }}$ & 0.420 & $<0.001^{\text {*** }}$ \\
\hline
\end{tabular}

Two multiple liner regression models were provided. Percent excess weight loss $(\% \mathrm{EWL})$ was the independent variable, and $R^{2}$ was the percentage of $\%$ EWL explained by the whole model. $* P<0.05$; $* * P<0.01 ; * * * P<0.001$

\section{Preoperative LCN2, Waist Circumference, and HbAc1 Were Independent Predictors for Excellent Weight Loss}

Excellent weight loss was defined as an EWL $\geq 75 \% 1$ year after LSG surgery. Of the 58 subjects who underwent LSG surgery and were incorporated into a logistic regression analysis, 28 patients did not achieve excellent weight loss. In multiple analyses, as shown in Table 4 Model 1 (Nagelkerke $R^{2}=0.673$ ), lower waist circumference and higher serum LCN2 levels at baseline were independently associated with excellent weight loss after LSG surgery. After adjusting for preoperative $\mathrm{HbAc1}$ and TG, preoperative HbAc1 levels indicated an independent association with excellent weight loss (Model 2, Nagelkerke $R^{2}=0.739$ ). Therefore, preoperative waist circumference and serum LCN2 and HbAc1 levels might independently predict excellent weight loss 1-year post-operation in our cohort.

\section{A New P Index as a Predictive Model for Excellent Weight Reduction}

Based on our findings, we identified waist circumference and preoperative LCN2 as the most predictive parameters for excellent weight reduction 12-month post-surgery in our dataset. Both variables achieved acceptable performance in ROC analyses with optimal cutoffs for waist circumference at $117.647 \mathrm{~cm}$ (sensitivity $80.00 \%$, specificity $85.70 \%$ ) and LCN2 level at $103.15 \mathrm{ng} / \mathrm{mL}$ (sensitivity $76.70 \%$, specificity $71.40 \%$ ), respectively (Table 5 and Fig. 3).

A new index, $\mathrm{P}$, was defined by Model 2 (Table 5 and Fig. 3). The new equation was developed as follows: $\mathrm{P}$ 
Table 4 Variables significantly affecting a response of more than $75 \%$ excess weight loss after LSG surgery

\begin{tabular}{|c|c|c|c|c|}
\hline & \multicolumn{2}{|l|}{ Model 1} & \multicolumn{2}{|l|}{ Model 2} \\
\hline & \multicolumn{2}{|l|}{ Nagelkerke $R^{2}=0.673$} & \multicolumn{2}{|l|}{ Nagelkerke $R^{2}=0.739$} \\
\hline & $O R(95 \% C I)$ & $P$ value & $O R(95 \% C I)$ & $P$ value \\
\hline Age & $0.979(0.910,1.053)$ & 0.563 & $0.983(0.905,1.067)$ & 0.681 \\
\hline Sex (female vs. male) & $0.248(0.022,2.851)$ & 0.263 & $0.118(0.007,2.077)$ & 0.144 \\
\hline Body mass index & $0.910(0.702,1.179)$ & 0.475 & $0.987(0.720,1.353)$ & 0.933 \\
\hline Waist circumference & $0.837(0.726,0.966)$ & $0.015^{*}$ & $0.766(0.625,0.940)$ & $0.011^{*}$ \\
\hline Lipocalin-2 & $1.055(1.019,1.092)$ & $0.003^{* *}$ & $1.076(1.028,1.126)$ & $0.002^{* *}$ \\
\hline $\mathrm{HbAc1}$ & - & - & $0.477(0.239,0.952)$ & $0.036^{*}$ \\
\hline Triglycerides & - & - & $1.761(0.917,3.383)$ & 0.089 \\
\hline
\end{tabular}

Excellent weight loss and not excellent were defined as EWL $\geq 75 \%$ and $\mathrm{EWL}<75 \%$, respectively. Adjusted odds ratio $(O R)$ and $95 \%$ confidence interval $(C I)$ for the probability of excellent weight loss 1 year after LSG surgery were given. $* P<0.05 ; * * P<0.01$; *** $P<0.001$ index $=(-0.017 \times$ age $($ years $)-2.134 \times$ sex $-0.014 \times$ BMI $\left(\mathrm{kg} / \mathrm{m}^{2}\right)-0.266 \times$ waist circumference $(\mathrm{cm})+0.073 \times \mathrm{LCN} 2$ $(\mathrm{ng} / \mathrm{mL})+0.566 \times$ triglycerides $(\mathrm{mmol} / \mathrm{L})-0.741 \times \mathrm{HbAc} 1$ $(\%)+33.018)$, with sex referred to as 1 in men and 2 in women. The AUC of the new P index was 0.949, significantly larger than those of LCN2 and waist circumference, indicating that the new $\mathrm{P}$ index is more reliable for predicting 1-year weight reduction after the operation. The optimum cutoff point for the new $\mathrm{P}$ index at 0.649 predicted excellent weight loss 12-month post-surgery with a sensitivity of $86.70 \%$ and specificity of $96.40 \%$, both parameters were higher than those achieved with waist circumference or serum LCN2 alone.

\section{Discussion}

Bariatric surgery is the only practical and effective intervention alternative for most patients with extreme obesity to achieve weight loss [19]. However, the effectiveness of weight loss varies among patients. According to Reinhold's classification [17, 18], success was regarded as $\geq 50 \%$ EWL, while excellent weight loss was defined when the \%EWL was $\geq 75 \%$. Some physiological characteristics of preoperative patients have been studied to predict success (\%EWL $\geq 50 \%)$ after bariatric surgery, such as sex, age, BMI, waist circumference, type II diabetes, dyslipidemia, hypertension, and smoking, with a rough similarity but lack of consensus [20]. Although $\geq 75 \%$ EWL was associated with better metabolic syndrome remission 1 year after bariatric surgery [21], there are few published studies regarding predictors of excellence (\%EWL $\geq 75 \%)$ after bariatric surgery, LCN2 is closely associated with obesity and obesity-related metabolic disorders, but its impact on weight loss after surgery is unknown. Our study aimed to ascertain whether preoperative serum LCN2 or any other factors independently predict excellence (\%EWL $\geq 75 \%)$ in weight reduction after LSG surgery.

Our previous observations indicated that patients with obesity had elevated serum LCN2 levels [8, 9]. In our study, excellent weight loss (\%EWL $\geq 75 \%)$ at 1 -year follow-up after LSG surgery was independently correlated with higher preoperative LCN2 levels. In addition, \% TWL was also

Table 5 Receiver-operating characteristic (ROC) for lipocalin-2, waist circumference, and the new P index as predictors for a surgery response of $\geq 75 \%$ EWL 1 year after LSG surgery

\begin{tabular}{lllllllll}
\hline & $\begin{array}{l}\text { Optimized } \\
\text { cutoff value }\end{array}$ & Sensitivity (\%) & Specificity (\%) & Youden index & AUC & $\begin{array}{l}\text { Standard } \\
\text { error (SE) }\end{array}$ & $95 \%$ CI & $P$ value \\
\hline New index P & 0.649 & 86.700 & 96.400 & 0.831 & 0.949 & 0.029 & $0.893-1.000$ & $<0.001^{* * *}$ \\
Waist circumference, cm & 117.647 & 80.000 & 85.700 & 0.657 & 0.865 & 0.050 & $0.767-0.962$ & $<0.001^{* * *}$ \\
Lipocalin-2, ng/mL & 103.150 & 76.700 & 71.400 & 0.481 & 0.715 & 0.070 & $0.578-0.853$ & $0.005^{* *}$ \\
\hline
\end{tabular}

New P index was calculated by the formula: $-0.017 \times$ age (years) $-2.134 \times \mathrm{sex}-0.014 \times \mathrm{BMI}\left(\mathrm{kg} / \mathrm{m}^{2}\right)-0.266 \times \mathrm{WC}(\mathrm{cm})+0.073 \times \mathrm{LCN} 2(\mathrm{ng} /$ $\mathrm{mL})+0.566 \times$ triglycerides $(\mathrm{mmol} / \mathrm{L})-0.741 \times \mathrm{HbAc} 1(\%)+33.018]$. Values were presented as 1 in male sex and 2 in female sex, and WC as waist circumference.

$A U C$ area under the curve, $95 \% C I$ 95\% confidence interval.

$* P<0.05 ; * * P<0.01 ; * * * P<0.001$ 


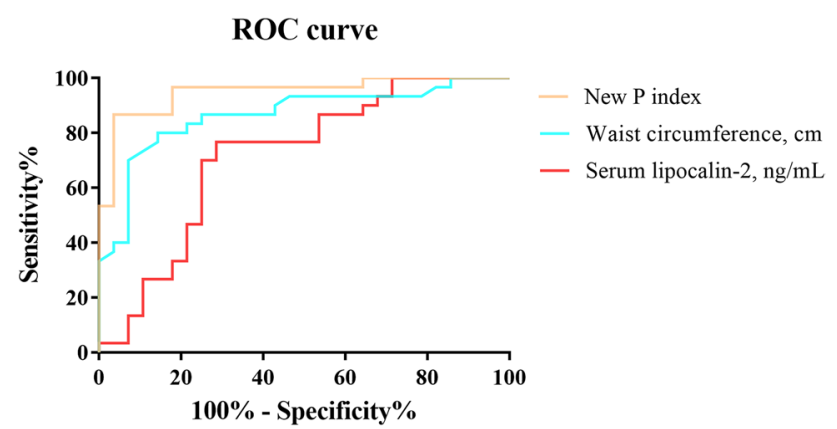

Fig. 3 Receiver operating characteristic (ROC) curve for serum lipocalin-2 (red line), waist circumference (blue line), and new $\mathrm{P}$ index (orange line) respectively in the prediction of a surgery response of $\geq 75 \%$ EWL 1 year after LSG surgery

significantly associated with serum LCN2, indicating that patients with higher preoperative LCN2 levels tend to lose more initial weight and are more likely to reach their ideal weight. Our study suggests that elevated serum LCN2 levels in patients with obesity might serve as a biomarker or even play an important role in weight reduction after LSG surgery. In a previous study, the ablation of LCN2 profoundly impaired adaptive thermogenesis through suppression of brown adipose tissue activity [22-24]. Circulating LCN2 suppresses food intake by activating the melanocortin-4 receptor signaling pathway in the hypothalamus [25]. LCN2 seems to be a key factor linking energy intake and energy expenditure with obesity, partly explaining the excellent weight reduction in patients with higher LCN2 at baseline. However, an in-depth understanding of these mechanisms remains to be elucidated.

In our cohort, a cross-sectional study showed that serum LCN2 levels were associated with several adiposity variables, including BMI, weight, waist circumference, and hip circumference, which concurs with a previous study in 229 adults, suggesting that increased fat mass might partly account for elevated serum LCN2 levels in humans with obesity [26]. Furthermore, we found a significantly negative association between serum LCN2 levels and several variables correlated with adverse lipid profiles. In agreement with the animal data, our findings implied that LCN2 might be a protective factor against lipid metabolic dysregulation $[22,27]$.

In addition to preoperative serum LCN2 levels, we found that waist circumference and $\mathrm{HbAc} 1$ levels at baseline were independent predictors for \%EWL. The effects of BMI and waist circumference remain controversial in predicting the success of bariatric surgery studies [28-30]. We found that $\%$ EWL was negatively associated with waist circumference but not with BMI in multiple regression analysis considering BMI and waist circumference together. In binary logistic regression analysis, Model 2 showed that $\mathrm{HbAc1}$ was an independent predictor of excellence after LSG surgery, consistent with a study by Ortega et al. [30].

Previous bariatric surgery studies have focused on the success of surgery (defined as \%EWL $\geq 50 \%$ or mean minus $1 \mathrm{SD}$ ) and performed a multiple logistic regression analysis including variables such as anthropometric and metabolic index, surgery style, and surgery access to predict weight reduction after bariatric surgery [28, 30-32]. In addition, several novel predictors (e.g., serum asprosin, phase angle, and apnea-hypopnea index) of weight reduction efficacy have emerged in recent years, but none of them, when combined with other parameters, provide a predictive model or display significance in a model $[7,15,20$, $33,34]$. To our knowledge, this is the first study to focus on preoperative predictors of excellent weight reduction (defined as \%EWL $\geq 75 \%$ ) after LSG surgery, pointing to better weight reduction and better metabolic relief. In our study, we determined Model 2 as a new P index considering serum LCN2 and other variables including sex, age, adiposity, and glucolipid metabolism. The optimum cutoff of $\mathrm{P}$ of 0.649 showed a high sensitivity of $86.70 \%$ and specificity of $96.40 \%$ for predicting excellent weight reduction post-surgery.

Our study had a few limitations. The main limitation is the relatively small single-center dataset, which limited the statistical power of the results, as well as the lack of a validation cohort. Second, the follow-up period was relatively short (1 year), and the follow-up rate at 3 months is only $64.9 \%$. Additionally, because the cohort included only Chinese individuals, any findings may not be generalizable to other ethnic groups. Additionally, we cannot exclude the effect of any unmeasured bias or confounding factors. Future prospective studies may provide more reliable information on the prediction of post-surgery weight loss. We have proposed a non-validated prediction model that needs to be confirmed in a large prospective study. The problem of excellent weight loss is multifactorial, and its resolution will require more research in the future to uncover more predictors to provide an optimal prediction model.

\section{Conclusion}

In this study, we have provided a better model and a new $\mathrm{P}$ index based on preoperative serum LCN2 and other parameters as effective predictors for excellent weight reduction during the 1-year postoperative follow-up after bariatric surgery. Our model and the proposed index still require future studies to confirm their external validity, clinical applicability, and generalizability. 
Acknowledgements We would like to thank Editage (www.editage.cn) for English language editing.

Funding Grant support: This work was supported by the Tranditional Medicine Project of Shanghai Municipal Health Commission (grant number 2020JP013), Shanghai Natural Science Foundation (grant number 21ZR1449400), and Shanghai Pujiang Talent Plan (grant number 18PJD038).

\section{Declarations}

Ethics Approval. The study was approved by the ethics committee of Shanghai Tenth People's Hospital. All procedures performed in studies involving human participants were in accordance with the ethical standards of the institutional research committee and with the 1964 Helsinki Declarations and its later amendments or comparable ethical standards.

Informed Consent. Informed consent was obtained from all individual participant included in the study.

Conflict of Interest The authors declare no competing interests.

Open Access This article is licensed under a Creative Commons Attribution 4.0 International License, which permits use, sharing, adaptation, distribution and reproduction in any medium or format, as long as you give appropriate credit to the original author(s) and the source, provide a link to the Creative Commons licence, and indicate if changes were made. The images or other third party material in this article are included in the article's Creative Commons licence, unless indicated otherwise in a credit line to the material. If material is not included in the article's Creative Commons licence and your intended use is not permitted by statutory regulation or exceeds the permitted use, you will need to obtain permission directly from the copyright holder. To view a copy of this licence, visit http://creativecommons.org/licenses/by/4.0/.

\section{References}

1. Gastroenterology TL, Obesity H. another ongoing pandemic. lancet Gastroenterol hepatol. 2021;6(6):411.

2. Maggard-Gibbons M, Maglione M, Livhits M, Ewing B, Maher $\mathrm{AR}, \mathrm{Hu}$ J, et al. Bariatric surgery for weight loss and glycemic control in nonmorbidly obese adults with diabetes: a systematic review. JAMA. 2013;309(21):2250-61.

3. Müller-Stich BP, Senft JD, Warschkow R, Kenngott HG, Billeter AT, Vit G, et al. Surgical versus medical treatment of type 2 diabetes mellitus in nonseverely obese patients: a systematic review and meta-analysis. Ann Surg. 2015;261(3):421-9.

4. Panagiotou OA, Markozannes G, Adam GP, Kowalski R, Gazula A, Di M, et al. Comparative effectiveness and safety of bariatric procedures in medicare-eligible patients: a systematic review. JAMA surgery. 2018;153(11):e183326.

5. O'Brien PE, Hindle A, Brennan L, Skinner S, Burton P, Smith A, et al. Long-term outcomes after bariatric surgery: a systematic review and meta-analysis of weight loss at 10 or more years for all bariatric procedures and a single-centre review of 20-year outcomes after adjustable gastric banding. Obes Surg. 2019;29(1):3-14.

6. Sjöström L, Peltonen M, Jacobson P, Sjöström CD, Karason K, Wedel $\mathrm{H}$, et al. Bariatric surgery and long-term cardiovascular events. JAMA. 2012;307(1):56-65.
7. Borges-Canha M, Neves JS, Mendonça F, Silva MM, Costa C, P $\mathrm{MC}$, et al. Beta cell function as a baseline predictor of weight loss after bariatric surgery. Frontiers endocrinol. 2021;12:714173.

8. Mosialou I, Shikhel S, Luo N, Petropoulou PI, Panitsas K, Bisikirska B, et al. Lipocalin-2 counteracts metabolic dysregulation in obesity and diabetes. The Journal of experimental medicine. 2020;217(10).

9. Wen X, Zhu B, Zhang Y, Mei F, Cheng X, Qian C, et al. Alterations in fat mass and bone mineral density are associated with decreased lipocalin-2 after laparoscopic sleeve gastrectomy in obese Chinese women. Obes Surg. 2019;29(9):2862-8.

10. Meyers K, López M, Ho J, Wills S, Rayalam S, Taval S. Lipocalin-2 deficiency may predispose to the progression of spontaneous age-related adiposity in mice. Sci Rep. 2020;10(1):14589.

11. Petropoulou PI, Mosialou I, Shikhel S, Hao L, Panitsas K, Bisikirska B, et al. Lipocalin-2 is an anorexigenic signal in primates. eLife. 2020;9.

12. Robert M, Espalieu P, Pelascini E, Caiazzo R, Sterkers A, Khamphommala L, et al. Efficacy and safety of one anastomosis gastric bypass versus Roux-en-Y gastric bypass for obesity (YOMEGA): a multicentre, randomised, open-label, non-inferiority trial. Lancet (London, England). 2019;393(10178):1299-309.

13. Ke Z, Li F, Gao Y, Tan D, Sun F, Zhou X, et al. The use of visceral adiposity index to predict diabetes remission in low BMI Chinese patients after bariatric surgery. Obes Surg. 2021;31(2):805-12.

14. Rebelos E, Moriconi D, Scalese M, Denoth F, Molinaro S, Siciliano $\mathrm{V}$, et al. Impact of postprandial hypoglycemia on weight loss after bariatric surgery. Obes Surg. 2020;30(6):2266-73.

15. Wang CY, Lin TA, Liu KH, Liao CH, Liu YY, Wu VC, et al. Serum asprosin levels and bariatric surgery outcomes in obese adults. Int j obesity (2005). 2019;43(5):1019-25.

16. Zhou B. [Predictive values of body mass index and waist circumference to risk factors of related diseases in Chinese adult population]. Zhonghua liu xing bing xue za zhi = Zhonghua liuxingbingxue zazhi. 2002;23(1):5-10.

17. Carandina S, Soprani A, Zulian V, Cady J. Long-term results of one anastomosis gastric bypass: a single center experience with a minimum follow-up of 10 years. Obes Surg. 2021;31(8):3468-75.

18. Reinhold RB. Critical analysis of long term weight loss following gastric bypass. Surgery, gynecology \& obstetrics. 1982;155(3):385-94.

19. Buchwald H, Avidor Y, Braunwald E, Jensen MD, Pories W, Fahrbach K, et al. Bariatric surgery: a systematic review and metaanalysis. JAMA. 2004;292(14):1724-37.

20. Gerken ALH, Rohr-Kräutle KK, Weiss C, Seyfried S, Reissfelder C, Vassilev G, et al. Handgrip strength and phase angle predict outcome after bariatric surgery. Obes Surg. 2021;31(1):200-6.

21. Tu Y, Pan Y, Han J, Pan J, Zhang P, Jia W, et al. A total weight loss of $25 \%$ shows better predictivity in evaluating the efficiency of bariatric surgery. Int j obesity (2005). 2021;45(2):396-403.

22. Guo H, Jin D, Zhang Y, Wright W, Bazuine M, Brockman DA, et al. Lipocalin-2 deficiency impairs thermogenesis and potentiates diet-induced insulin resistance in mice. Diabetes. 2010;59(6):1376-85.

23. Guo H, Bazuine M, Jin D, Huang MM, Cushman SW, Chen X. Evidence for the regulatory role of lipocalin 2 in high-fat dietinduced adipose tissue remodeling in male mice. Endocrinology. 2013;154(10):3525-38.

24. Zhang Y, Guo H, Deis JA, Mashek MG, Zhao M, Ariyakumar D, et al. Lipocalin 2 regulates brown fat activation via a nonadrenergic activation mechanism. J Biol Chem. 2014;289(32):22063-77.

25. Mosialou I, Shikhel S, Liu JM, Maurizi A, Luo N, He Z, et al. MC4R-dependent suppression of appetite by bone-derived lipocalin 2. Nature. 2017;543(7645):385-90.

26. Wang Y, Lam KSL, Kraegen EW, Sweeney G, Zhang J, Tso AW, et al. Lipocalin-2 is an inflammatory marker closely associated 
with obesity, insulin resistance, and hyperglycemia in humans. Clin Chem. 2007;53(1):34-41.

27. Deis JA, Guo H, Wu Y, Liu C, Bernlohr DA, Chen X. Adipose lipocalin 2 overexpression protects against age-related decline in thermogenic function of adipose tissue and metabolic deterioration. Molecular metabolism. 2019;24:18-29.

28. Barhouch AS, Padoin AV, Casagrande DS, Chatkin R, Süssenbach SP, Pufal MA, et al. Predictors of excess weight loss in obese patients after gastric bypass: a 60-month follow-up. Obes Surg. 2016;26(6):1178-85.

29. Menenakos E, Stamou KM, Albanopoulos K, Papailiou J, Theodorou D, Leandros E. Laparoscopic sleeve gastrectomy performed with intent to treat morbid obesity: a prospective single-center study of 261 patients with a median follow-up of 1 year. Obes Surg. 2010;20(3):276-82.

30. Ortega E, Morínigo R, Flores L, Moize V, Rios M, Lacy AM, et al. Predictive factors of excess body weight loss 1 year after laparoscopic bariatric surgery. Surg Endosc. 2012;26(6):1744-50.
31. Coupaye M, Sabaté JM, Castel B, Jouet P, Clérici C, Msika S, et al. Predictive factors of weight loss 1 year after laparoscopic gastric bypass in obese patients. Obes Surg. 2010;20(12):1671-7.

32. Sisik A, Basak F. Presurgical predictive factors of excess weight loss after laparoscopic sleeve gastrectomy. Obes Surg. 2020;30(8):2905-12.

33. de Raaff CA, Coblijn UK, de Vries N, Heymans MW, van den Berg BT, van Tets WF, et al. Predictive factors for insufficient weight loss after bariatric surgery: does obstructive sleep apnea influence weight loss? Obes Surg. 2016;26(5):1048-56.

34. Neves JS, Souteiro P, Oliveira SC, Pedro J, Magalhães D, Guerreiro $\mathrm{V}$, et al. Preoperative thyroid function and weight loss after bariatric surgery. Int j obesity (2005). 2019;43(2):432-6.

Publisher's Note Springer Nature remains neutral with regard to jurisdictional claims in published maps and institutional affiliations. 